\title{
Lifestyle Factors and Metabolic Syndrome among Workers: The Role of Interactions between Smoking and Alcohol to Nutrition and Exercise
}

\author{
Jui-Hua Huang ${ }^{1,+}{ }^{,}$Ren-Hau Li ${ }^{2,+}$, Shu-Ling Huang ${ }^{2,3,+}$, Hon-Ke Sia ${ }^{4}$, Yu-Ling Chen ${ }^{1}$ and \\ Feng-Cheng Tang ${ }^{5,6, *}$ \\ Received: 11 October 2015; Accepted: 11 December 2015; Published: 16 December 2015 \\ Academic Editor: Paul B. Tchounwou \\ 1 Occupational Health Center, Changhua Christian Hospital, Changhua 500, Taiwan; \\ Juihua1025@gmail.com (J.-H.H.); qaz5410@yahoo.com.tw (Y.-L.C.) \\ 2 Department of Psychology, Chung-Shan Medical University, Taichung 402, Taiwan; \\ davidrhlee@yahoo.com.tw (R.-H.L.); shuling@csmu.edu.tw (S.-L.H.) \\ 3 Room of Clinical Psychology, Chung-Shan Medical University Hospital, Taichung 402, Taiwan \\ 4 Division of Endocrinology and Metabolism, Changhua Christian Hospital, Changhua 500, Taiwan; \\ 90279@cch.org.tw \\ 5 Department of Occupational Medicine, Changhua Christian Hospital, Changhua 500, Taiwan \\ 6 Department of Leisure Services Management, Chaoyang University of Technology, Taichung 413, Taiwan \\ * Correspondence: 106159@cch.org.tw; Tel.: +886-4-728-6929; Fax: +886-4-727-0910 \\ + These authors contributed equally to this work.
}

\begin{abstract}
This study aimed to investigate (1) relations of smoking and alcohol to metabolic syndrome (MetS) and its components, with nutrition and exercise controlled; and (2) interactions between smoking/alcohol and nutrition/exercise on MetS. This cross-sectional study enrolled 4025 workers. Self-reported lifestyles, anthropometric values, blood pressure (BP), and biochemical determinations were obtained. Among males, smoking significantly increased the risk of low high-density lipoprotein cholesterol (HDL-C), high triglyceride, abdominal obesity (AO), and MetS. Additionally, smoking showed significant interaction effects with nutrition on high BP, AO, and MetS; after further analysis, nutrition did not decrease above-mentioned risks for smokers. However, there was no significant interaction of smoking with exercise on any metabolic parameter. Alcohol increased the risk of AO, but decreased low HDL-C. It also showed an interaction effect with exercise on $\mathrm{AO}$; after further analysis, exercise decreased $\mathrm{AO}$ risk for drinkers. Among females, alcohol significantly decreased the risk of high fasting blood glucose, but did not show significant interaction with nutrition/exercise on any metabolic parameter. In conclusion, in males, smoking retained significant associations with MetS and its components, even considering benefits of nutrition; exercise kept predominance on lipid parameters regardless of smoking status. Alcohol showed inconsistencies on metabolic parameters for both genders.
\end{abstract}

Keywords: alcohol; health promotion; metabolic syndrome; nutrition and exercise behaviors; smoking; worker

\section{Introduction}

The metabolic syndrome (MetS) is a clustering of abdominal obesity, elevated blood pressure (BP), low serum high-density lipoprotein cholesterol (HDL-C) levels, elevated serum triglycerides (TG), and impaired fasting blood glucose (FBG) [1]. MetS and its components, such as dyslipidemia and hypertension, increase the risk of cardiovascular disease [2]. The most important causes of MetS are aging, unhealthy diet, and sedentary lifestyle [3,4]. The characteristics of work, including 
job types, stress, and working hours are also associated with MetS [5-7]. Employees of several categories are therefore considered as a high-risk population in the development of MetS. In Taiwan, workplace health-promoting programs have been proposed to prevent the development of MetS. These strategies focus mainly on promoting workers' physical activity and healthy eating [8].

Nutrition and exercise behaviors are important factors in the prevention of MetS and its components [3,9]. Lutsey et al. suggested that consumption of a Western dietary pattern, meat and fried foods promotes the incidence of MetS [3]. Reading nutrition labels showed an association with reducing the risk of MetS [10]. In addition, regular leisure-time physical activities prevent unhealthy weight gain [11]. Low level leisure physical activity raised the odds ratios of MetS [12]. Dietary and exercise behaviors were associated with obesity-specific quality of life and work productivity as well [13]. These studies stated that nutrition and exercise behaviors were confirmedly related to MetS and its components.

Smoking and excessive alcohol drinking habits can also be contributing factors to developing MetS [14,15]. Cigarette smoking was considered to be related to MetS [16]. The smoking habit may increase the risk of lower HDL-C, higher TG, and abdominal obesity [17]. In addition, a higher drinking quantity and frequent binge drinking are associated with a higher prevalence of MetS, and are gender-specific [18]. Yoon et al. reported that alcohol consumption was inversely associated with the risk of low HDL-C, but showed an increasing dose-response relation between alcohol drinking and the risk of MetS [19]. In general, smoking and drinking habits are related to higher prevalence of MetS [20].

The individual influence of nutrition, exercise, smoking, and alcohol on the progression of MetS has been well studied, but little is known about the interactions of these lifestyle factors on the MetS and its components. A previous study indicated that smoking might have different detrimental effects on lipid-related cardiovascular disease in people with different grades of physical activity [21]. Breslow et al. also reported that alcohol-drinking patterns were associated with diet quality [22]. The associations between smoking/alcohol habits and nutrition/exercise behaviors may occur. However, only few investigators have analyzed the interactive effects of these lifestyle factors on the risk of MetS and its components in detail. Previous research stressed these interactive effects mainly on vascular diseases, such as stroke [23,24]; but studies regarding their influences on MetS were comparatively neglected. In Taiwan, a national survey in 2012 showed that about $32.7 \%$ of men and $4.3 \%$ of women were smokers. In addition, approximately $1.82 \%$ of the young adults and $4.82 \%$ of the middle-aged adults drank alcohol on a daily basis [25]. These issues should be considered. We thus investigated a series of lifestyle factors to clarify their interactions on MetS and its components among Taiwanese workers.

The interactive relationships among smoking, alcohol consumption, nutrition, exercise, and MetS were complicated. Further clarification of these associations is required. We proposed the hypotheses: (1) the associations between smoking or alcohol and MetS were positively significant, with nutrition and exercise controlled; (2) the interactions between smoking/alcohol and nutrition/exercise on MetS were significant as well. The aims of this study were: (1) to investigate the relations of smoking and alcohol habits to MetS itself and its components, when taking nutrition and exercise health behaviors into consideration; and (2) to explore the interactions between smoking/alcohol habits and nutrition/exercise behaviors on the development of MetS among male and female workers in Taiwan.

\section{Methods}

\subsection{Study Design}

This study was conducted in 2012 using a cross-sectional research method with convenient sampling. Workers aged 20 years or over, from four companies, were recruited for the study. All of the participants took part in the study voluntarily. Personal information, nutrition, and 
exercise behaviors were obtained by a self-reported questionnaire. Blood biochemical data, such as FBG, TG, and HDL-C, and anthropometric measurements were collected by tests performed in medical laboratories and non-invasive physical examination. The present study was a component of the Taiwan Workplace Health Promotion Scheme, and was approved by an independent ethics committee, the Institutional Review Board of the Changhua Christian Hospital (Taiwan).

\subsection{Assessment of Nutrition and Exercise Health Behaviors}

The data on nutrition and exercise behaviors were obtained using the subscales of Health-Promoting Lifestyle Profile II [26]. Nutrition behavior included nine items, and exercise behavior included eight items. These items were translated into traditional Chinese characters for the participants. In the present survey, the subscales of nutrition and exercise behaviors in Chinese have shown acceptable internal consistency, with Cronbach's alpha of 0.78 and 0.85 , respectively. Participants were asked to rate each item on a four-point Likert scale (1. Never, 2. Sometimes, 3. Often, and 4. Routinely). The mean score of each subscale, ranging from 1 to 4 , was calculated from the total subscale scores divided by the number of response items. A higher score indicates a greater level of participation in health-promoting behaviors.

\subsection{Assessment of Smoking and Alcohol Drinking Habits}

Information regarding smoking and alcohol drinking habits was collected from each question. Participants were asked to tick their statuses of smoking and alcohol drinking separately. Smoking habits were classified as following three conditions: non-smoking, occasional smoking, and daily smoking. For statistical purposes, workers who smoked occasionally or daily were classified as workers who smoked. In addition, workers were classified as non-alcohol drinking workers and alcohol drinking workers (including occasional and daily alcohol drinking). Of all the workers who smoked in this study, only seven of them were female. Therefore, the associations between smoking and dependent variables were not analyzed for the females.

\subsection{Metabolic Parameters and Definition of MetS}

MetS is defined as a co-occurrence of three out of five of the following medical conditions: elevated FBG, low HDL-C levels, high serum TG, elevated BP, and abdominal (central) obesity, according to the guidelines of the National Cholesterol Education Program [1]. According to the definition of the Health Promotion Administration, Ministry of Health and Welfare in Taiwan [27], $\mathrm{FBG} \geqslant 100 \mathrm{mg} / \mathrm{dL}$ is considered as an impaired level. Ideal blood lipid levels are recommended as HDL-C $\geqslant 50 \mathrm{mg} / \mathrm{dL}$ among females and $\geqslant 40 \mathrm{mg} / \mathrm{dL}$ among males. TG $<150 \mathrm{mg} / \mathrm{dL}$ is optimal. Systolic BP $<130$ and diastolic BP $<85$ are defined as normal levels of BP. Waist circumference (WC) $\geqslant 90 \mathrm{~cm}$ for men and $\geqslant 80 \mathrm{~cm}$ for women are considered as abdominal obesity. However, in the present study, the definition of MetS did not consider medical treatment or history of the disease.

\subsection{Statistical Analysis}

For statistical purposes, workers who smoked or drank alcohol were coded as 1 and non-smoking or non-drinking workers were coded as 0 . Workers aged 40 or over were coded as 1 and workers aged under 40 were coded as 0 . For categorical variables in the contingency table, data were presented in number (n) and percent (\%). The chi-square test was used to examine the differences in smoking status, alcohol status and whether with MetS by gender. This test was also used to examine the relationships between smoking/alcohol status and MetS and each metabolic parameter (e.g., $\mathrm{FBG}<100 / \geqslant 100$ ). For continuous variables, data were presented in mean $\pm \mathrm{SD}$, and comparisons of the means were analyzed by two-tailed $t$-test (two groups). Additionally, multiple logistic regression model with the "Enter" method was used to examine the individual relations of smoking and alcohol habits to MetS and its components separately for men and women; furthermore, interaction terms for lifestyle factors were added to this model to predict health outcomes. In this model, nutrition 
and exercise were first centered at their means respectively and then to form interaction terms with smoke or alcohol. This multiple logistic regression model comprised dependent variables including MetS and five metabolic parameters, and independent variables including age, lifestyle factors (smoking, alcohol drinking, nutrition, and exercise behaviors), and interaction terms for lifestyle factors. The analysis for women did not include smoking and interaction terms with it due to the very small number of female smokers. Data were presented with odds ratio (OR) and $95 \%$ confidence interval for OR (95\% CIs). All statistical procedures were performed using SPSS 17.0 statistical software (SPSS Inc., Chicago, IL, USA); a $p$-value less than 0.05 was considered statistically significant.

\section{Results}

\subsection{Comparing the Characteristics of the Workers by Gender}

In the present study, 5096 workers, aged 20 or over, were recruited as subjects from the four companies. Data with more than $25 \%$ of question items uncompleted were excluded to ensure the credibility of the results. In total, a final number of 4025 participants were enrolled. The average age of the participants was $43.1 \pm 10.0$ in the study. The comparisons of nutrition and exercise behaviors, metabolic parameters, MetS, smoking and alcohol of the participants by gender are summarized in Table 1. Male workers had significantly higher FBG, TG, systolic, and diastolic BP, WC, and percentage of MetS than the females did $(p<0.001)$. In addition, there were indications of gender differences in lifestyle factors, including nutrition behavior, smoking and alcohol habits $(p<0.001)$. Male workers had a lower nutrition behavior score, and higher percentages in cigarette smoking and alcohol drinking compared to the females.

Table 1. Comparing the characteristics of the workers by gender.

\begin{tabular}{|c|c|c|c|c|}
\hline Variables & Total $(n=4025)$ & Male $(n=3294)$ & Female $(n=731)$ & \\
\hline Continuous variables (Mean \pm SD) & & & & $p$ for $t$-test \\
\hline Age $(\mathrm{y})$ & $43.1 \pm 10.0$ & $42.6 \pm 10.3$ & $45.6 \pm 7.6$ & $<0.001$ \\
\hline Exercise behavior score & $1.96 \pm 0.56$ & $1.96 \pm 0.56$ & $1.96 \pm 0.54$ & 0.884 \\
\hline Nutrition behavior score & $2.47 \pm 0.44$ & $2.43 \pm 0.43$ & $2.66 \pm 0.43$ & $<0.001$ \\
\hline \multicolumn{5}{|l|}{ Metabolic parameters } \\
\hline FBG $(\mathrm{mg} / \mathrm{dL})$ & $93.4 \pm 18.8$ & $94.2 \pm 19.6$ & $89.7 \pm 14.6$ & $<0.001$ \\
\hline HDL-C (mg/dL) & $52.8 \pm 12.6$ & $50.8 \pm 11.7$ & $61.3 \pm 13.0$ & $<0.001$ \\
\hline $\mathrm{TG}(\mathrm{mg} / \mathrm{dL})$ & $133.7 \pm 101.1$ & $139.1 \pm 105.4$ & $109.1 \pm 74.2$ & $<0.001$ \\
\hline Systolic BP (mmHg) & $123.1 \pm 15.5$ & $124.5 \pm 14.9$ & $116.6 \pm 16.7$ & $<0.001$ \\
\hline Diastolic BP (mmHg) & $78.7 \pm 11.3$ & $79.1 \pm 11.3$ & $76.9 \pm 10.9$ & $<0.001$ \\
\hline $\mathrm{WC}(\mathrm{cm})$ & $82.0 \pm 9.5$ & $83.9 \pm 8.7$ & $73.6 \pm 8.4$ & $<0.001$ \\
\hline Categorical variables (n (\%)) & & & & $p$ for chi-square test \\
\hline \multicolumn{5}{|l|}{ MetS } \\
\hline$<3$ components & $3347(83.2)$ & $2698(81.9)$ & $649(88.8)$ & $<0.001$ \\
\hline$\geqslant 3$ components & $677(16.8)$ & $595(18.1)$ & $82(11.2)$ & \\
\hline \multicolumn{5}{|l|}{ Smoking } \\
\hline with & $905(22.3)$ & $898(27.0)$ & $7(1.0)$ & $<0.001$ \\
\hline without & $3150(77.7)$ & 2425 (73.0) & 725 (99.0) & \\
\hline \multicolumn{5}{|l|}{ Alcohol } \\
\hline with & $1833(45.2)$ & $1694(51.0)$ & $139(19.0)$ & $<0.001$ \\
\hline without & $2218(54.8)$ & $1627(49.0)$ & $591(81.0)$ & \\
\hline
\end{tabular}

Notes: FBG: fasting blood glucose; HDL-C: high-density lipoprotein cholesterol; TG: triglyceride; BP: blood pressure; WC: waist circumference; MetS: metabolic syndrome.

\subsection{Comparing Metabolic Parameters by Smoking and Alcohol Habits}

Differences in metabolic parameters by smoking and alcohol habits are shown in Table 2. The workers with smoking had a higher proportion of low HDL-C $(21.9 \%$ vs. $14.1 \%, p<0.001)$, high TG $(36.4 \%$ vs. $25.5 \%, p<0.001)$ and MetS $(22.0 \%$ vs. $15.3 \%, p<0.001)$, when compared with the non-smoking workers. In addition, the workers who drank alcohol had a higher proportion of high FBG $(19.7 \%$ vs. $17.0 \%, p=0.023)$, high TG $(29.8 \%$ vs. $26.4 \%, p=0.016)$, and increased 
WC $(24.4 \%$ vs. $21.2 \%, p=0.016)$, when compared with the workers who did not drink alcohol. The difference of MetS by alcohol drinking had a marginal tendency toward statistical significance $(p=0.053)$.

Table 2. Comparing metabolic parameters by smoking and alcohol habits.

\begin{tabular}{|c|c|c|c|c|c|c|}
\hline \multirow[t]{2}{*}{ Variables } & \multicolumn{2}{|c|}{ Smoking } & \multicolumn{4}{|c|}{ Alcohol Drinking } \\
\hline & With, n (\%) & Without, n (\%) & $p$ & With, n (\%) & Without, n (\%) & $p$ \\
\hline \multicolumn{7}{|l|}{ FBG (mg/dL) } \\
\hline$\geqslant 100$ & $182(20.3)$ & $549(17.6)$ & & $358(19.7)$ & $374(17.0)$ & \\
\hline $\mathrm{n}$ & 895 & 3128 & & 1814 & 2205 & \\
\hline \multicolumn{7}{|l|}{ HDL-C $(\mathrm{mg} / \mathrm{dL})$} \\
\hline $\mathrm{n}$ & 905 & 3144 & & 1830 & 2215 & \\
\hline \multicolumn{7}{|l|}{ TG (mg/dL) } \\
\hline$<150$ & $576(63.6)$ & $2346(74.5)$ & $<0.001$ & $1287(70.2)$ & $1633(73.6)$ & 0.016 \\
\hline$\geqslant 150$ & $329(36.4)$ & $804(25.5)$ & & $546(29.8)$ & $585(26.4)$ & \\
\hline $\mathrm{n}$ & 905 & 3150 & & 1833 & 2218 & \\
\hline \multicolumn{7}{|l|}{$\mathrm{WC}(\mathrm{cm})$} \\
\hline$<90$ for men or $<80$ for women & $685(75.7)$ & $2453(77.9)$ & 0.167 & $1386(75.6)$ & $1748(78.8)$ & 0.016 \\
\hline$\geqslant 90$ for men or $\geqslant 80$ for women & $220(24.3)$ & $697(22.1)$ & & $447(24.4)$ & $470(21.2)$ & \\
\hline $\mathrm{n}$ & 905 & 3150 & & 1833 & 2218 & \\
\hline \multicolumn{7}{|l|}{ MetS } \\
\hline$<3$ components & $698(78.0)$ & $2643(84.7)$ & $<0.001$ & $1484(81.9)$ & $1855(84.2)$ & 0.053 \\
\hline$\geqslant 3$ components & $197(22.0)$ & $479(15.3)$ & & $327(18.1)$ & $347(15.8)$ & \\
\hline $\mathrm{n}$ & 895 & 3122 & & 1811 & 2202 & \\
\hline
\end{tabular}

Notes: FBG: fasting blood glucose; HDL: high-density lipoprotein; TG: triglycerides; BP: blood pressure; WC: waist circumference; MetS: metabolic syndrome.

\subsection{Interactions between Smoking/Alcohol Habits and Exercise/Nutrition Behaviors on the Risk of Metabolic Parameters}

The results of smoking and alcohol habits and their separate interactions with nutrition and exercise behaviors in relation to the risks of metabolic parameters are shown in Table 3.

For male participants, a smoking habit showed higher risks in all of metabolic parameters. The risks even reached a significant level in low HDL-C (OR $=2.01,95 \%$ CI 1.61-2.52, $p<0.001)$, high TG $(\mathrm{OR}=1.62,95 \%$ CI 1.35-1.94, $p<0.001)$, and increased WC (OR = 1.22, 95\% CI 1.01-1.49, $p=0.042$ ). In addition, the interaction effect of a smoking habit and nutrition behavior on high BP showed a statistical significance $(\mathrm{OR}=1.06,95 \% \mathrm{CI} 1.00-1.11, p=0.033)$. Meaning, nutrition behavior on the risk of developing high BP for smoking male workers relative to that of non-smoking male workers was 1.06. For male non-smoking workers, nutrition behavior significantly decreased the risk of elevated BP $(\mathrm{OR}=0.97,95 \%$ CI 0.94-1.00, $p=0.036)$. After further analysis, nutrition behavior did not significantly decrease the risk of high $\mathrm{BP}$ for male workers with smoking $(\mathrm{OR}=1.06 \times 0.97=1.03$, 95\% CI 0.94-1.11, $p=0.512$ ). The interaction effect of smoking and nutrition on increased WC $(\mathrm{OR}=1.08,95 \%$ CI 1.02-1.14, $p=0.011)$ also showed significant results. For non-smoking workers, the OR of nutrition behavior for increased WC was 0.99. The OR was not significantly different from unity. After further analysis for workers with smoking, nutrition behavior was observed to constitute a non-significant risk of experiencing increased WC $(\mathrm{OR}=1.08 \times 0.99=1.07$, 95\% CI $0.98-1.17, p=0.079$ ). Smoking habit did not show any significant interaction with exercise behavior on metabolic parameters, though exercise was observed to have individual significant effect on reducing the risk of low HDL-C (OR $=0.95,95 \%$ CI 0.92-0.99, $p=0.010)$ and high TG $(\mathrm{OR}=0.96,95 \% \mathrm{CI}$ $0.93-0.98, p=0.002)$. It implies exercise behavior kept the predominant benefits on lipid parameters regardless of smoking status. 
Table 3. Interactions between smoking/alcohol habits and exercise/nutrition behaviors on the risk of metabolic parameters.

\begin{tabular}{|c|c|c|c|c|c|c|c|c|c|c|}
\hline \multirow[t]{2}{*}{ Variables } & \multirow{2}{*}{$\begin{array}{c}\text { FBG (mg/dL) } \\
\geqslant 100 \\
\text { OR }(95 \% \text { CI })\end{array}$} & \multirow{2}{*}{\multicolumn{2}{|c|}{$\begin{array}{c}\text { HDL-C }(\mathrm{mg} / \mathrm{dL}) \\
<40 \text { for Men or } \\
<50 \text { for Women } \\
\text { OR }(95 \% \mathrm{CI}) \\
\end{array}$}} & \multicolumn{3}{|c|}{$\begin{array}{c}\mathrm{TG}(\mathrm{mg} / \mathrm{dL}) \\
\geqslant 150\end{array}$} & \multirow{2}{*}{$\begin{array}{c}\text { BP }(\mathrm{mm} \mathrm{Hg}) \\
\text { SBP } \geqslant 130 \text { and } \\
\text { DBP } \geqslant 85 \\
\text { OR }(95 \% \mathrm{CI})\end{array}$} & \multicolumn{3}{|c|}{$\begin{array}{l}W C(\mathrm{~cm}) \geqslant 90 \\
\text { for Men or } \geqslant 80 \\
\text { for Women }\end{array}$} \\
\hline & & & & $p$ & OR (95\% CI) & $p$ & & $p$ & OR (95\% CI) & $p$ \\
\hline \multicolumn{11}{|l|}{ Man } \\
\hline Constant & $0.20(0.17-0.24)$ & $<0.001$ & $0.22(0.19-0.27)$ & $<0.001$ & $0.28(0.24-0.33)$ & $<0.001$ & $0.41(0.35-0.47)$ & $<0.001$ & $0.21(0.18-0.25)$ & $<0.001$ \\
\hline Age $(\geqslant 40 /<40)$ & $1.24(0.03-1.49)$ & 0.021 & $0.55(0.44-0.67)$ & $<0.001$ & $1.76(1.50-2.07)$ & $<0.001$ & $2.99(2.56-3.48)$ & $<0.001$ & $1.42(1.20-1.70)$ & $<0.001$ \\
\hline Nutrition & $1.00(0.97-1.04)$ & 0.891 & $1.03(0.98-1.07)$ & 0.215 & $1.01(0.98-1.04)$ & 0.568 & $0.97(0.94-1.00)$ & 0.036 & $0.99(0.96-1.03)$ & 0.765 \\
\hline Exercise & $0.99(0.95-1.02)$ & 0.354 & $0.95(0.92-0.99)$ & 0.010 & $0.96(0.93-0.98)$ & 0.002 & $1.02(1.00-1.05)$ & 0.115 & $0.97(0.94-1.00)$ & 0.090 \\
\hline Smoke (with/without) & $1.06(0.86-1.31)$ & 0.589 & $2.01(1.61-2.52)$ & $<0.001$ & $1.62(1.35-1.94)$ & $<0.001$ & $1.09(0.92-1.31)$ & 0.318 & $1.22(1.01-1.49)$ & 0.042 \\
\hline Alcohol (with/without) & $1.19(1.00-1.42)$ & 0.056 & $0.72(0.58-0.89)$ & 0.002 & $0.98(0.84-1.14)$ & 0.777 & $1.06(0.92-1.23)$ & 0.431 & $1.22(1.03-1.44)$ & 0.023 \\
\hline Smoke $\times$ Nutrition & $1.00(0.94-1.06)$ & 0.872 & $1.05(0.98-1.12)$ & 0.157 & $1.02(0.97-1.08)$ & 0.377 & $1.06(1.00-1.11)$ & 0.033 & $1.08(1.02-1.14)$ & 0.011 \\
\hline Smoke $\times$ Exercise & $1.00(0.96-1.05)$ & 0.651 & $1.01(0.95-1.06)$ & 0.833 & $0.98(0.94-1.02)$ & 0.369 & $0.99(0.94-1.03)$ & 0.474 & $1.05(1.00-1.10)$ & 0.060 \\
\hline Alcohol $\times$ Nutrition & $1.02(0.96-1.07)$ & 0.560 & $0.97(0.91-1.03)$ & 0.301 & $0.98(0.94-1.03)$ & 0.472 & $1.02(0.98-1.07)$ & 0.284 & $1.03(0.98-1.08)$ & 0.283 \\
\hline Alcohol $\times$ Exercise & $1.00(0.96-1.05)$ & 0.847 & $1.01(0.96-1.07)$ & 0.708 & $1.02(0.98-1.06)$ & 0.456 & $0.98(0.95-1.02)$ & 0.324 & $0.95(0.91-0.99)$ & 0.018 \\
\hline \multicolumn{11}{|l|}{ Woman } \\
\hline Constant & $0.06(0.03-0.13)$ & $<0.001$ & $0.22(0.14-0.34)$ & $<0.001$ & $0.12(0.07-0.22)$ & $<0.001$ & $0.13(0.07-0.22)$ & $<0.001$ & $0.21(0.13-0.33)$ & $<0.001$ \\
\hline Age $(\geqslant 40 /<40)$ & $3.06(1.28-7.29)$ & 0.012 & $1.14(0.71-1.83)$ & 0.601 & $1.84(1.02-3.32)$ & 0.044 & $4.07(2.30-7.20)$ & $<0.001$ & $1.53(0.94-2.51)$ & 0.091 \\
\hline Nutrition & $0.94(0.88-1.01)$ & 0.076 & $1.02(0.96-1.07)$ & 0.578 & $0.94(0.88-1.00)$ & 0.036 & $0.98(0.93-1.03)$ & 0.341 & $0.94(0.89-0.99)$ & 0.023 \\
\hline Exercise & $1.05(0.99-1.12)$ & 0.125 & $0.99(0.94-1.04)$ & 0.727 & $0.99(0.94-1.05)$ & 0.828 & $0.99(0.94-1.03)$ & 0.581 & $0.99(0.94-1.040$ & 0.561 \\
\hline Alcohol (with/without) & $0.21(0.07-0.65)$ & 0.006 & $1.17(0.71-1.92$ & 0.540 & $0.74(0.40-1.37)$ & 0.337 & $0.75(0.46-1.21)$ & 0.239 & $0.92(0.56-1.51)$ & 0.739 \\
\hline Alcohol $\times$ Nutrition & $1.17(0.92-1.47)$ & 0.196 & $0.97(0.86-1.10)$ & 0.675 & $1.02(0.87-1.19)$ & 0.815 & $1.00(0.88-1.13)$ & 0.983 & $1.00(0.87-1.15)$ & 0.993 \\
\hline Alcohol $\times$ Exercise & $0.89(0.72-1.10)$ & 0.277 & $0.96(0.85-1.07)$ & 0.423 & $0.87(0.75-1.02)$ & 0.084 & $1.02(0.92-1.14)$ & 0.668 & $0.94(0.84-1.07)$ & 0.355 \\
\hline
\end{tabular}

Notes: FBG: fasting blood glucose (Including 3284 male and 729 female subjects in this multiple logistic regression); HDL-C: high-density lipoprotein cholesterol (3309 male and 730 female study subjects); TG: triglyceride (3315 male and 730 female study subjects); BP: blood pressure (3315 male and 730 female study subjects); WC: waist circumference (3315 male and 730 female study subjects). 
In male workers, alcohol drinking significantly increased the risk of increased WC $(\mathrm{OR}=1.22$, 95\% CI 1.03-1.44, $p=0.023)$, but it decreased the risk of low HDL-C (OR $=0.72,95 \%$ CI $0.58-0.89$, $p=0.002$ ). In addition, the interaction effect of alcohol drinking habit and exercise behavior on increased WC displayed a statistical significance (OR $=0.95,95 \%$ CI $0.91-0.99, p=0.018$ ). For workers who did not drink alcohol, exercise behavior did not significantly decrease the risk of increased WC; however, exercise behavior significantly decreased the risk of increased WC $(\mathrm{OR}=0.95 \times 0.97=0.92$, $95 \%$ CI $0.86-0.99, p=0.004)$ for workers with alcohol drinking. Compared with workers who did not drink alcohol, exercise behavior significantly decreased the risk of increased WC in workers with alcohol drinking. Besides, alcohol habits did not show any significant interaction with nutrition behavior on any metabolic parameter.

For female participants, alcohol drinking on reducing high FBG reached a significant level $(\mathrm{OR}=0.21,95 \% \mathrm{CI} 0.07-0.65, p=0.006)$. However, alcohol drinking did not show significant interaction effects with nutrition or exercise behavior on the risk of metabolic parameters.

\subsection{Interaction between Smoking/Alcohol Habits and Exercise/Nutrition Behaviors on the Risk of MetS}

The results of smoking and alcohol habits and their separate interactions with nutrition and exercise behaviors in relation to the risks of MetS are shown in Table 4.

For male participants, a smoking habit showed a significantly higher risk in MetS (OR $=1.64$, 95\% CI 1.33-2.02, $p<0.001$ ). The interaction effect of smoking and nutrition on MetS (OR $=1.07$, 95\% CI 1.01-1.14, $p=0.028$ ) also showed a significant result. For non-smoking workers, the OR of nutrition behavior for MetS was 0.96. After further analysis for workers who smoked, nutrition behavior showed a non-significant risk of MetS (OR $=1.07 \times 0.96=1.03, p=0.357)$. In addition, a smoking habit did not show any significant interaction with exercise behavior on MetS. On the other hand, an alcohol habit did not show any significant interaction with nutrition behavior on MetS for male participants.

For female participants, alcohol drinking did not show significant interaction effects with nutrition or exercise behavior on the risk of MetS. The analysis for women did not include smoking due to the very small number of female participants with smoking.

Table 4. Interactions between smoking/alcohol habits and exercise/nutrition behaviors on the risk of MetS.

\begin{tabular}{ccc}
\hline Variables & MetS & $p$ \\
\cline { 2 - 2 } & OR (95\% CI) & \\
Man & & \\
Constant & $0.13(0.11-0.16)$ & $<0.001$ \\
Age $(\geqslant 40 /<40)$ & $1.79(1.47-2.17)$ & $<0.001$ \\
Nutrition & $0.96(0.93-1.00)$ & 0.068 \\
Exercise & $0.98(0.94-1.01)$ & 0.170 \\
Smoke (with/without) & $1.64(1.33-2.02)$ & $<0.001$ \\
Alcohol (with/without) & $1.06(0.88-1.28)$ & 0.530 \\
Smoke $\times$ Nutrition & $1.07(1.01-1.14)$ & 0.028 \\
Smoke $\times$ Exercise & $1.00(0.95-1.05)$ & 0.977 \\
Alcohol $\times$ Nutrition & $1.05(0.99-1.11)$ & 0.125 \\
Alcohol $\times$ Exercise & $0.98(0.94-1.03)$ & 0.498 \\
\hline Woman & & \\
Constant & $0.07(0.04-0.14)$ & $<0.001$ \\
Age $(\geqslant 40 /<40)$ & $2.37(1.14-4.96)$ & 0.021 \\
Nutrition & $0.90(0.84-0.97)$ & 0.005 \\
Exercise & $0.98(0.92-1.05)$ & 0.598 \\
Alcohol $($ with $/$ without $)$ & $0.67(0.33-1.39)$ & 0.284 \\
Alcohol $\times$ Nutrition & $0.98(0.79-1.20)$ & 0.822 \\
Alcohol $\times$ Exercise & $0.89(0.73-1.08)$ & 0.233 \\
\hline
\end{tabular}

Note: MetS: metabolic syndrome (Including 3315 male and 730 female subjects in this multiple logistic regression). 


\section{Discussion}

The present study provides evidence of lifestyle interactions with regard to MetS and its components among Taiwanese workers. Statistical analysis of the smoking habit was carried out for only male participants because the number of female participants who smoked was small. The results in this study found that smoking increased the risk of MetS and its components among male workers. Particularly, there were significant associations with HDL-C, TG, WC and MetS. Moreover, smoking showed interaction effects with nutrition behavior on BP, WC, and MetS. However, alcohol drinking showed inconsistent associations with MetS and its components, regardless whether they were males or females. For male workers, an alcohol habit increased the risk of increased WC, but decreased the risk of low HDL-C. In addition, alcohol showed an interaction effect with exercise behavior on WC. For female workers, an alcohol habit significantly decreased the risk of high FBG. However, alcohol drinking did not have a significant interaction effect with exercise or nutrition behavior on the risk of MetS.

Smoking may raise sympathetic activity and increase the circulating cortisol, catecholamines, vasopressin, and growth hormone levels [28,29]. Therefore, smoking has been considered as playing a causal role in the development of MetS [29]. Slagter et al. reported that smoking was significantly associated with lower HDL-C, higher TG, and abdominal obesity [17]. Calo et al. also reported that there was an increased risk of MetS among current smokers, especially those with excess consumption [30]. Indeed, our findings, which indicated that smoking increased the risk of low HDL-C, high TG, abdominal obesity, and MetS in male workers, were consistent with the results in other studies.

In the present study, nutrition health behavior significantly decreased the risk of elevated BP in male workers non-smoking. However, the interaction effect of smoking and nutrition behavior was significant with a higher odd ratio on elevated BP. That is to say, nutrition behavior among smoking and non-smoking males had different associations with developing elevated BP. In addition, the result of further analysis for male workers who smoked showed that nutrition behavior did not significantly decrease the risk of high BP. In previous research, consumption of an unhealthy diet was associated with elevated BP [31]. Conversely, good nutrition behavior showed an association in reducing the risk of high BP [32]. Furthermore, smoking was also found to be an important risk factor for developing high BP [33]. Our results implied that the benefit of nutrition behavior might not surpass the adverse effects of smoking in regard to the impact on high BP in male workers. A smoking habit should therefore be taken into consideration when designing workplace health promotion programs to prevent the development of elevated BP. The mechanism of the interaction of smoking and nutrition behavior on BP also deserves further study.

The influences of alcohol drinking on the components of MetS exhibit both positive and negative results [34]. The association between alcohol drinking and development of MetS is stronger among red wine drinkers due to polyphenoles contained in red wine. Polyphenoles may raise the activity of endothelial nitric oxide synthase (eNOS), which plays a key role in the development of MetS. Promoting eNOS activity, which involves the transport of HDL-C, may elucidate the mechanism of the elevated plasma levels of HDL-C in response to alcohol. Conversely, downregulating eNOS activity may result in the incidence of insulin resistance, high BP, and abnormal blood lipid levels [34]. Yoon et al. reported that alcohol consumption had a negative association with the risk of low HDL-C, but also that there was an increasing dose-response relation between alcohol consumption and the risk of MetS [19]. In addition, research exploring gender-specific relationships between alcohol patterns and the prevalence of MetS has been carried out. Lee indicated that excessive drinking frequency was dose-dependently associated with elevated TG, hyperglycemia, elevated BP, and abdominal obesity in men, and with hyperglycemia and elevated BP in women [18]. Another study showed that higher alcohol consumption raised the risk of abdominal obesity in men only [35]. Our data also showed that the associations between alcohol drinking and components of MetS were controversial and gender differential. In this study, an alcohol habit decreased the risk of low HDL-C, 
and increased the risk of abdominal obesity in male workers; alcohol habit decreased the risk of high FBG in female workers.

The interaction between alcohol and either nutrition or exercise behavior was seldom discussed in previous studies. The amount and type of alcohol consumed was considered as playing important roles in enhancing or inhibiting the development of MetS [36,37]. A recent study showed medium-high risk alcohol consumption mainly affected males, and was associated with increased risk of hypertension; among females, low risk consumption was associated with a lower prevalence of certain components of the MetS, including obesity and hypertension [38]. Wang et al. also reported that light to moderate alcohol consumption was found to be protective regarding the risks of obesity or being overweight among females [39]. In our study, the interaction effects of alcohol drinking and either nutrition or exercise behavior on MetS and its components were not significant, except for WC among male workers. More detailed information regarding the amount and type of alcohol consumed is recommended for relevant research in the future. Notably, the benefit of exercise behavior can be found in the impact on WC in alcohol drinking male workers. For this group of workers, to prevent the development of abdominal obesity, exercise behavior should therefore be taken into consideration when designing workplace health promotion program.

Females usually have a lower rate of alcohol drinking or smoking than males in Taiwan and several countries $[25,38,40]$. A national survey in Taiwan showed that approximately $29.6 \%$ of male employees and $3.1 \%$ of female employees were smokers; the occurrences of alcohol drinking were $37.8 \%$ for male employees and $13.3 \%$ for female employees. Also, the prevalence of MetS among male workers was significantly higher than among female workers in this study. In particular, the relations of smoking and alcohol drinking to the development of MetS were gender-specific. Therefore, gender difference should be an issue when establishing policy to prevent MetS and in designing workplace health-promoting programs.

Although this study has provided some insights into the interaction effects of nutrition/exercise and smoking/alcohol on the risk of MetS and its components, it has several limitations. First, there was about $21 \%$ of missing data in the present study. We were not sure whether they were missing at random (MAR). The results should therefore be interpreted carefully. Second, the assessment of lifestyle was highly dependent on the self-reported questionnaire. Overestimation, underestimation, or poor recall may produce confounded results. Third, our analyses did not consider frequency, consumption, and specific types of alcohol. Fourthly, the definition of metabolic syndrome did not consider the history of the disease or the effectiveness of medical treatment, and thus the classification of metabolic syndrome may have a bias. This study focused on the participants' current state of MetS. Finally, it was a cross-sectional study and could not establish the causal direction of the impacts of smoking and alcohol habits on the risk of developing MetS and its components. The inference of the findings should therefore be applied with caution. Further studies adopting a longitudinal study design, with objective tools to more accurately assess frequency, quantity, and types of alcohol consumption, as well as lifestyle factors are recommended.

\section{Conclusions}

Male workers with smoking habits showed higher risks on MetS and its components. Moreover, smoking habits showed a significant interaction effect with nutrition behavior on the risk of high BP, abdominal obesity, and MetS in male workers, though its interaction with exercise was not significant. In general, taking the benefits of nutrition behavior into further consideration, smoking habits still retained significant relations with MetS and its components. Exercise kept the predominant benefits on lipid parameters regardless of smoking status. Alcohol drinking showed inconsistent associations with the development of MetS for both males and females, although, for male workers with alcohol habits, maintaining an exercise routine could be tried to decrease the risk of abdominal obesity. It is therefore recommended that workplace health promotion practitioners should develop more specific strategies to target male workers with smoking habit or poor exercise behavior. 
Acknowledgments: This study was supported by Grant No.1001205V from the Health Promotion Administration, Ministry of Health and Welfare, Taiwan. We especially thank the workers who participated in the present study and provided valuable information. The authors also thank Fu-Chou Cheng for his comments on data interpretation.

Author Contributions: The authors' contributions were as follows: Conception and design of the study: Jui-Hua Huang, Shu-Ling Huang, Yu-Ling Chen, and Feng-Cheng Tang. Data collection and processing: Jui-Hua Huang and Yu-Ling Chen. Statistical analysis: Jui-Hua Huang, Shu-Ling Huang, and Ren-Hau Li. Interpretation of data: Jui-Hua Huang, Shu-Ling Huang, Ren-Hau Li, Hon-Ke Sia, and Feng-Cheng Tang. Drafting the manuscript: Jui-Hua Huang, Shu-Ling Huang, Ren-Hau Li, Hon-Ke Sia, and Feng-Cheng Tang. Obtaining funding: Feng-Cheng Tang. All authors were involved in preparing the outline of the manuscript, making comments on the manuscript, and approval the final version of the article.

Conflicts of Interest: The authors declare no conflict of interest.

\section{References}

1. Alexander, C.M.; Landsman, P.B.; Teutsch, S.M.; Haffner, S.M. NCEP-defined metabolic syndrome, diabetes, and prevalence of coronary heart disease among NHANES III participants age 50 years and older. Diabetes 2003, 52, 1210-1214. [CrossRef] [PubMed]

2. Isomaa, B.; Almgren, P.; Tuomi, T.; Forsen, B.; Lahti, K.; Nissen, M.; Taskinen, M.R.; Groop, L. Cardiovascular morbidity and mortality associated with the metabolic syndrome. Diabetes Care 2001, 24, 683-689. [CrossRef] [PubMed]

3. Lutsey, P.L.; Steffen, L.M.; Stevens, J. Dietary intake and the development of the metabolic syndrome-The Atherosclerosis Risk in Communities study. Circulation 2008, 117, 754-761. [CrossRef] [PubMed]

4. Churilla, J.R.; Fitzhugh, E.C. Total physical activity volume, physical activity intensity, and metabolic syndrome: 1999-2004 National Health and Nutrition Examination Survey. Metab. Syndr. Relat. Disord. 2012, 10, 70-76. [CrossRef] [PubMed]

5. Almadi, T.; Cathers, I.; Chow, C.M. Associations among work-related stress, cortisol, inflammation, and metabolic syndrome. Psychophysiology 2013, 50, 821-830. [CrossRef] [PubMed]

6. Kwon, C.S.; Lee, J.H. The Association between type of work and insulin resistance and the metabolic syndrome in middle-aged Korean men: Results from the Korean national health and nutrition examination survey IV (2007 2009). World J. Mens Health 2013, 31, 232-238. [CrossRef] [PubMed]

7. Violanti, J.M.; Burchfiel, C.M.; Hartley, T.A.; Mnatsakanova, A.; Fekedulegn, D.; Andrew, M.E.; Charles, L.E.; Vila, B.J. Atypical work hours and metabolic syndrome among police officers. Arch. Environ. Occup. Health 2009, 64, 194-201. [CrossRef] [PubMed]

8. Sia, H.K.; Su, Y.C.; Tang, F.C. Workplace health promotion in practice for metabolic syndrome. Ind. Saf. Hyg. 2012, 276, 38-42. (In Chinese)

9. Lackland, D.T.; Voeks, J.H. Metabolic syndrome and hypertension: Regular exercise as part of lifestyle management. Curr. Hypertens. Rep. 2014, 16, 1-7. [CrossRef] [PubMed]

10. Kang, H.T.; Shim, J.Y.; Lee, Y.J.; Linton, J.A.; Park, B.J.; Lee, H.R. Reading nutrition labels is associated with a lower risk of metabolic syndrome in Korean adults: The 2007-2008 Korean NHANES. Nutr. Metab. Cardiovasc. Dis. 2013, 23, 876-882. [CrossRef] [PubMed]

11. Haskell, W.L.; Lee, I.M.; Pate, R.R.; Powell, K.E.; Blair, S.N.; Franklin, B.A.; Macera, C.A.; Heath, G.W.; Thompson, P.D.; Bauman, A. Physical activity and public health: Updated recommendation for adults from the American College of Sports Medicine and the American Heart Association. Med. Sci. Sports Exerc. 2007, 39, 1423-1434. [CrossRef] [PubMed]

12. Choi, M.; Yeom, H.A.; Jung, D. Association between physical activity and metabolic syndrome in older adults in Korea: Analysis of data from the Korean National Health and Nutrition Examination Survey IV. Nurs. Health Sci. 2013, 15, 379-386. [CrossRef] [PubMed]

13. Cash, S.W.; Beresford, S.A.; Henderson, J.A.; McTiernan, A.; Xiao, L.; Wang, C.Y.; Patrick, D.L. Dietary and physical activity behaviours related to obesity-specific quality of life and work productivity: Baseline results from a worksite trial. Br. J. Nutr. 2012, 108, 1134-1142. [CrossRef] [PubMed]

14. Corwin, E.J.; McCoy, C.S.; Whetzel, C.A.; Ceballos, R.M.; Klein, L.C. Risk indicators of metabolic syndrome in young adults: A preliminary investigation on the influence of tobacco smoke exposure and gender. Heart Lung 2006, 35, 119-129. [CrossRef] [PubMed] 
15. Lee, K.W.; Park, B.J.; Kang, H.T.; Lee, Y.J. Alcohol-drinking patterns and metabolic syndrome risk: The 2007 Korean National Health and Nutrition Examination Survey. Alcohol 2011, 45, 499-505. [CrossRef] [PubMed]

16. Oh, S.W.; Yoon, Y.S.; Lee, E.S.; Kim, W.K.; Park, C.; Lee, S.; Jeong, E.K.; Yoo, T. Association between cigarette smoking and metabolic syndrome: The Korea National Health and Nutrition Examination Survey. Diabetes Care 2005, 28, 2064-2066. [CrossRef] [PubMed]

17. Slagter, S.N.; van Vliet-Ostaptchouk, J.V.; Vonk, J.M.; Boezen, H.M.; Dullaart, R.P.; Kobold, A.C.; Feskens, E.J.; van Beek, A.P.; van der Klauw, M.M.; Wolffenbuttel, B.H. Associations between smoking, components of metabolic syndrome and lipoprotein particle size. BMC Med. 2013, 11. [CrossRef] [PubMed]

18. Lee, K. Gender-specific relationships between alcohol drinking patterns and metabolic syndrome: The Korea National Health and Nutrition Examination Survey 2008. Public Health Nutr. 2012, 15, 1917-1924. [CrossRef] [PubMed]

19. Yoon, Y.S.; Oh, S.W.; Baik, H.W.; Park, H.S.; Kim, W.Y. Alcohol consumption and the metabolic syndrome in Korean adults: The 1998 Korean National Health and Nutrition Examination Survey. Am. J. Clin. Nutr. 2004, 80, 217-224. [PubMed]

20. Yu, M.; Xu, C.X.; Zhu, H.H.; Hu, R.Y.; Zhang, J.; Wang, H.; He, Q.F.; Su, D.T.; Zhao, M.; Wang, L.X.; et al. Associations of cigarette smoking and alcohol consumption with metabolic syndrome in a male Chinese population: A cross-sectional study. J. Epidemiol. 2014, 24, 361-369. [CrossRef] [PubMed]

21. Katano, H.; Ohno, M.; Yamada, K. Protection by physical activity against deleterious effect of smoking on carotid intima-media thickness in young Japanese. J. Stroke Cerebrovasc. Dis. 2013, 22, 176-183. [CrossRef] [PubMed]

22. Breslow, R.A.; Guenther, P.M.; Smothers, B.A. Alcohol drinking patterns and diet quality: The 1999-2000 National Health and Nutrition Examination Survey. Am. J. Epidemiol. 2006, 163, 359-366. [CrossRef] [PubMed]

23. Evenson, K.R.; Rosamond, W.D.; Cai, J.; Toole, J.F.; Hutchinson, R.G.; Shahar, E.; Folsom, A.R. Physical activity and ischemic stroke risk. The atherosclerosis risk in communities study. Stroke 1999, 30, 1333-1339. [CrossRef] [PubMed]

24. Gillum, R.F.; Mussolino, M.E.; Ingram, D.D. Physical activity and stroke incidence in women and men. The NHANES I epidemiologic follow-up study. Am. J. Epidemiol. 1996, 143, 860-869.

25. Lin, C.Y.; Chen, K.H.; Chang, H.Y.; Tseng, F.Y.; Chen, C.Y. The relationship between the pattern of alcohol consumption and healthcare utilization in Taiwan. Taiwan J. Public Health Soc. Work 2014, 33, 197-208.

26. Walker, S.N.; Sechrist, K.R.; Pender, N.J. The health-promoting lifestyle profile: Development and psychometric characteristics. Nurs. Res. 1987, 36, 76-81. [CrossRef] [PubMed]

27. Health Promotion Administration, Ministry of Health and Welfare in Taiwan. Definition of Metabolic Symdrome in Adults (Aged $\geqslant 20$ Years) [2007 Taiwan]. Available online: http://www.hpa.gov.tw/ BHPNet/Web/HealthTopic/TopicArticle.aspx?No=200712250123\&parentid=023 (accessed on 26 January 2015).

28. Chiolero, A.; Faeh, D.; Paccaud, F.; Cornuz, J. Consequences of smoking for body weight, body fat distribution, and insulin resistance. Am. J. Clin. Nutr. 2008, 87, 801-809. [PubMed]

29. Balhara, Y.P. Tobacco and metabolic syndrome. Indian J. Endocrinol. Metab. 2012, 16, 81-87. [CrossRef] [PubMed]

30. Calo, W.A.; Ortiz, A.P.; Suarez, E.; Guzman, M.; Perez, C.M. Association of cigarette smoking and metabolic syndrome in a Puerto Rican adult population. J. Immigr. Minor. Health 2013, 15, 810-816. [CrossRef] [PubMed]

31. Hermansen, K. Diet, blood pressure and hypertension. Br. J. Nutr. 2000, 83, S113-S119. [CrossRef] [PubMed]

32. Appel, L.J.; Champagne, C.M.; Harsha, D.W.; Cooper, L.S.; Obarzanek, E.; Elmer, P.J.; Stevens, V.J.; Vollmer, W.M.; Lin, P.H.; Svetkey, L.P.; et al. Effects of comprehensive lifestyle modification on blood pressure control: Main results of the PREMIER clinical trial. JAMA 2003, 289, 2083-2093. [PubMed]

33. Halperin, R.O.; Gaziano, J.M.; Sesso, H.D. Smoking and the risk of incident hypertension in middle-aged and older men. Am. J. Hypertens. 2008, 21, 148-152. [CrossRef] [PubMed]

34. Jelski, W.; Szmitkowski, M. Effect of ethanol on metabolic syndrome. Pol. Arch. Med. Wewn. 2007, 117, 306-311. [PubMed] 
35. Schroder, H.; Morales-Molina, J.A.; Bermejo, S.; Barral, D.; Mandoli, E.S.; Grau, M.; Guxens, M.; de Jaime Gil, E.; Alvarez, M.D.; Marrugat, J.; et al. Relationship of abdominal obesity with alcohol consumption at population scale. Eur. J. Nutr. 2007, 46, 369-376. [CrossRef] [PubMed]

36. Hansel, B.; Thomas, F.; Pannier, B.; Bean, K.; Kontush, A.; Chapman, M.J.; Guize, L.; Bruckert, E. Relationship between alcohol intake, health and social status and cardiovascular risk factors in the Urban Paris-Ile-de-France Cohort: Is the cardioprotective action of alcohol a myth? Eur. J. Clin. Nutr. 2010, 64, 561-568. [CrossRef] [PubMed]

37. Klatsky, A.L. Alcohol and cardiovascular health. Physiol. Behav. 2010, 100, 76-81. [CrossRef] [PubMed]

38. Felix-Redondo, F.J.; Fernandez-Berges, D.; Palomo Cobos, L.; Buitrago Ramirez, F.; Perez Castan, J.F.; Lozano Mera, L. Prevalence alcohol consumption and cardiovascular risk factors in an Extremadura health area. Hermex study. Aten. Prim. 2012, 44, 201-208. [CrossRef] [PubMed]

39. Wang, L.; Lee, I.M.; Manson, J.E.; Buring, J.E.; Sesso, H.D. Alcohol consumption, weight gain, and risk of becoming overweight in middle-aged and older women. Arch. Intern. Med. 2010, 170, 453-461. [CrossRef] [PubMed]

40. Ng, M.; Freeman, M.K.; Fleming, T.D.; Robinson, M.; Dwyer-Lindgren, L.; Thomson, B.; Wollum, A.; Sanman, E.; Wulf, S.; Lopez, A.D.; et al. Smoking prevalence and cigarette consumption in 187 countries, 1980-2012. JAMA 2014, 311, 183-192. [CrossRef] [PubMed]

(C) 2015 by the authors; licensee MDPI, Basel, Switzerland. This article is an open access article distributed under the terms and conditions of the Creative Commons by Attribution (CC-BY) license (http:/ / creativecommons.org/licenses/by/4.0/). 